\title{
Índices de eficiência reprodutiva de búfalos da Amazônia Oriental do Brasil
}

\author{
[Indices of reproductive efficiency of buffaloes of the Brazilian Eastern Amazon]
}

\author{
R.N.C. Camargo Júnior ${ }^{1}$, J.R.F. Marques ${ }^{2}$, C.R. Marcondes $^{2}$, C.V. Araújo ${ }^{3}$, J.F. Aguiar ${ }^{4}$, \\ L.C. Marques ${ }^{5}$, A.E. Rodrigues ${ }^{5}$ \\ ${ }^{1}$ Instituto Federal de Educação, Ciência e Tecnologia do Pará - Santarém, PA \\ ${ }^{2}$ Embrapa Amazônia Oriental - Belém, PA \\ ${ }^{3}$ Universidade Federal do Mato Grosso - UFMT - Sinop, MT \\ ${ }^{4}$ Secretaria de Estado de Meio Ambiente - SEMA - Belém, PA \\ ${ }^{5}$ Aluna de pós-graduação - UPFA/Embrapa/UFRA - Belém, PA
}

\begin{abstract}
RESUMO
Foram avaliados registros de manejo reprodutivo de búfalos das raças Jafarabadi, Murrah, Mediterrâneo, Carabao e Tipo Baio, bem como seus mestiços, no período de 1983 a 2005. Estudaram-se as características reprodutivas: idade à primeira cria (média de 1052,52 $\pm 120,45$ dias), intervalo de partos

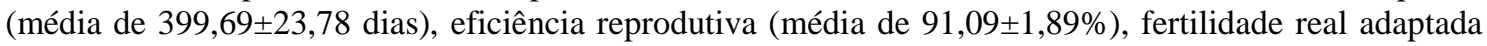

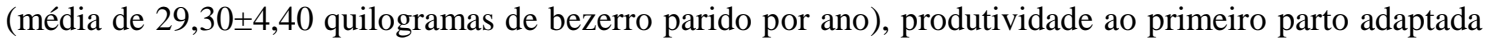
(média de 33,75 $\pm 6,89$ quilogramas) e produtividade acumulada adaptada (média de 22,86 $\pm 6,55$ quilogramas de bezerro parido por ano). Foram verificadas influências do ano de parto e o grau de sangue da fêmea sobre as características estudadas, o que indica que tais efeitos devem ser incluídos na avaliação genética e na seleção dos animais. Os índices produtivos utilizados mostraram-se de grande valia para a seleção, devendo ser mais estudadas suas interações relativas ao desempenho geral do rebanho.
\end{abstract}

Palavras-chave: Bubalus bubalis, manejo reprodutivo, seleção

\begin{abstract}
We evaluated records of reproductive management Jaffarabadi buffalo breeds, Murrah, Mediterranean, Carabao and Baio types, and their crosses, in the period from 1983 to 2005. We studied the following reproductive characteristics: Age at first calving (mean 1052.52 \pm 120.45 days), calving interval (399.69 \pm 23.78 mean days), Reproductive Efficiency (average 91.09 $\pm 1,89 \%)$, Real Adapted Fertility (average $29.30 \pm 4.40 \mathrm{~kg}$ calf calved each year), the First Labor Productivity Adapted (mean $33.75 \pm 6.89 \mathrm{~kg}$ ) and Accumulated Productivity Adapted (mean $22.86 \pm 6.55 \mathrm{~kg}$ calf calved each year). Influences were observed in the year of delivery and degree of blood on the female traits, suggesting that such effects should be included in genetic evaluation and selection of animals. The production indices used were of great value for the selection, and should be well studied for their interactions with the overall performance of the herd.
\end{abstract}

Keywords: Bubalus bubalis, reproductive management, selection

\section{INTRODUÇÃO}

A criação de búfalos vem se difundindo mundialmente devido à vantagem econômica que tal criação pode oferecer em relação à de outros ruminantes domésticos, principalmente pelo fato de os búfalos apresentarem características como rusticidade e adaptação às variadas condições climáticas e de manejo.

Recebido em 2 de dezembro de 2010

Aceito em 22 de maio de 2012

E-mail: camargojunior@gmail.com 
Segundo dados de 2003 do IBGE, o efetivo do rebanho bubalino no Pará aproxima-se das 500 mil cabeças, com crescimento de $22,5 \%$ entre 1998 e 2003. O estado possui 143 municípios, dentre os quais 16 encontram-se no Arquipélago de Marajó, onde estão localizados 50\% dos búfalos do Pará. Portanto, o Pará possui 50\% do rebanho bubalino brasileiro, e o Marajó possui $50 \%$ do rebanho do estado, logo conclui-se que o maior rebanho bubalino brasileiro está localizado no Marajó (Barbosa, 2005).

Em alguns momentos, detectou-se que um dos maiores problemas para os criadores de búfalos é o desconhecimento de alternativas de manejo e, principalmente, a falta de reprodutores selecionados que possibilitem o melhoramento genético dos rebanhos, sobretudo para leite A importância econômica na exploração desses animais reside, também, nas vantagens proporcionadas quanto à fertilidade, longevidade, eficiência de conversão alimentar e aptidão para a produção de leite, carne e para o trabalho.

Neste estudo objetivou-se estudar índices relacionados à eficiência do manejo reprodutivo de búfalos na Amazônia Oriental e os fatores que os influenciam, em suas diferentes raças e composições raciais.

\section{MATERIAL E MÉTODOS}

Utilizaram-se dados produtivos do período de 1983 a 2005. As informações referentes ao período de 1983 até o início de 1996 são oriundas dos rebanhos Jafarabadi, Murrah, Mediterrâneo, Carabao e Tipo Baio, bem como de seus mestiços, pertencentes ao Campo Experimental do Baixo Amazonas - CEBA, à margem esquerda do Rio Amazonas, município de Monte Alegre - Pará ( $2^{\circ} 00^{\prime} 30^{\prime}$ 'de latitude sul e $54^{\circ} 04^{\prime} 13$ " de longitude W. Gr), com uma altitude de $30 \mathrm{~m}$.

No período de junho de 1996 a 2005, os dados foram coletados no Campo Experimental do Marajó Emerson Salimos - CEMES, em Salvaterra, na Ilha de Marajó, onde o projeto Banco de Germoplasma Animal da Amazônia Oriental - BAGAM realiza estudos sobre conservação genética e desenvolve ações de manejo e produção da raça Carabao e Tipo Baio, criados em condições do trópico úmido amazônico.
Os dados foram codificados de acordo com Camargo Júnior (2007), que considerou as raças e/ou grupos genéticos, a ordem dos partos, o sexo das crias e as estações de parto, sendo estudadas as seguintes características de interesse econômico: eficiência reprodutiva (ER), fertilidade real adaptada (FR), produtividade ao primeiro parto adaptada (PP), produtividade acumulada adaptada (PA), idade à primeira cria (IPC) e intervalo de partos (IDP). Os dados foram trabalhados em planilhas do Microsoft ${ }^{\circledR}$ Office Excel e submetidos aos cálculos da estatística descritiva.

Para determinar os efeitos significativos sobre as características, utilizou-se o modelo estatístico: $Y_{i j k l}=\mu+G S F_{i}+A N O P_{j}+E P_{k}+e_{i j k l}$, em que: $\mu$ é a média geral; $\mathrm{GSF}_{\mathrm{i}}$ é o grau de sangue da fêmea; $\mathrm{ANOP}_{\mathrm{j}}$ é o ano do parto; $\mathrm{EP}_{\mathrm{k}}$ é a estação do parto; e $\mathrm{e}_{\mathrm{ijkl}}$ é o erro aleatório inerente a cada observação.

As análises de variância foram efetuadas por meio do PROC GLM do SAS (Statistical..., 2002), e a eficiência reprodutiva foi calculada pelos métodos apresentados por Marques (1991). Outro método utilizado para avaliar a produtividade da fêmea foi a FR (Lôbo, 1996), adaptada às informações disponíveis no Programa de Melhoramento Genético da Raça Nelore, a qual avaliou a fertilidade da vaca e sua habilidade materna. Este índice expressa quilogramas de bezerros nascidos por ano:

$F R=\frac{P B N \times 365}{I D P}$, em que:

$I D P=$ média dos intervalos de partos observados;

$P B N=$ peso do bezerro ao nascer;

$365=$ constante que permite expressar a fertilidade em base anual.

Para a avaliação de búfalas primíparas, considerou-se a PP, conforme proposto por Lôbo (1996):

$P P=\frac{P B N \times 36}{I P C}$, em que:

$P P=$ produtividade ao primeiro parto adaptada;

$P B N=$ peso do bezerro ao nascer;

$I P C=$ idade à primeira cria;

36 = idade à primeira cria média das matrizes estudadas, em meses. 
Outro índice estudado foi a PA, adaptada de Lôbo (1996). Este índice indica a produtividade da fêmea, em quilos de bezerros nascidos por ano. Mede a capacidade do animal em se reproduzir regularmente e a uma menor idade, bem como em parir animais com maior peso:

$$
P A=\frac{P_{n} \times n_{p} \times c_{a}}{I B P_{n}-c_{i}}, \text { em que: }
$$

$P_{n}$ = média do peso dos bezerros ao nascer;

$n_{p}=$ número total de bezerros paridos;

$c_{a}=$ constante igual a 365 dias, que permite expressar a fertilidade em base anual;
$I B P_{n}=$ idade da búfala por ocasião do último parto;

$c_{i}=$ constante igual a 706 dias, aproximadamente 23 meses, considerando-se a média da IPC dos animais observados.

\section{RESULTADOS E DISCUSSÃO}

Os índices de eficiência reprodutiva encontrados neste estudo, bem como suas fontes de variação, podem ser visualizados na Tab. 1 .

Tabela 1. Índices de eficiência reprodutiva de búfalos encontrados no período de 1983 a 2005

\begin{tabular}{ccccccccc} 
Índice & Registros $^{1}$ & Média $^{2}$ & \multicolumn{1}{c}{$\mathrm{DP}^{3}$} & $\mathrm{Min}^{4}$ & $\mathrm{Max}^{5}$ & $\mathrm{CV}^{6}$ & $\mathrm{R}^{2(7)}$ & $\mathrm{FV}^{8}$ \\
\hline IPC & 3275 & 1052,52 & $\pm 120,45$ & 737 & 1271 & 10,32 & 0,2598 & GSF \\
IDP & 792 & 399,69 & $\pm 23,78$ & - & - & 5,88 & 0,06593 & - \\
ER (\%) & 587 & 91,09 & $\pm 1,89$ & - & - & 2,05 & 0,0879 & - \\
FR & 809 & 29,30 & $\pm 4,40$ & 0,99 & 44,27 & 1189 & 0,2141 & GSF \\
PP & 337 & 33,75 & $\pm 6,89$ & 10 & 62 & 10,20 & 0,3342 & ANOP \\
PA & 588 & 22,86 & $\pm 6,55$ & 2,08 & 55,35 & 6,55 & 0,2548 & ANOP \\
GSF \\
\hline
\end{tabular}

${ }^{1}$ Número de registros analisados. ${ }^{2}$ Média geral encontrada, não ajustada. ${ }^{3}$ Desvio- padrão. ${ }^{4}$ Valor mínimo. ${ }^{5}$ Valor máximo. ${ }^{6}$ Coeficiente de variação (\%). ${ }^{7}$ Causas de variação. ${ }^{8}$ Fontes de Variação.

Sobre a IPC, De Franciscis (1979) reportou médias semelhantes à encontrada neste estudo, variando de 32 a 33 meses para idade ao primeiro parto. No entanto, Marques et al. (1985), para esta mesma característica, em animais das raças Murrah e Mediterrâneo bem como em mestiços dessas raças, encontraram média de 1.194,6 dias (39,16 meses), superior à encontrada na presente pesquisa. Baruselli (1997) desenvolveu diversos trabalhos referentes à IPC de novilhas da raça Murrah, encontrando uma média de $1.068,9$ dias ( $\pm 35,04$ meses), valor um pouco superior ao encontrado neste estudo. Cassiano et al. (2003) observaram que a média da IPC foi 1.088,03 dias ( \pm 35 meses), muito próxima à encontrada nesta pesquisa.

Por sua vez, Batista et al. (1994) obtiveram média de 36,2 meses para IPC. Rolim Filho (2006) encontrou o valor do IPC igual a $39,52 \pm 7,54$, com valores mínimo e máximo de 23,31 e 64,55 meses, respectivamente. Naquele estudo, em um total de 418 observações, a média da IPC foi $39,5 \pm 6,58 ; 39,6 \pm 9,85$ e $36,6 \pm 7,12$ meses para os sistemas de monta natural, inseminação artificial e inseminação artificial em tempo fixo, respectivamente.

O modelo proposto foi capaz de explicar aproximadamente $26 \%$ das causas de variação $\left(\mathrm{R}^{2}=0,2598\right)$. O GSF e o ANOP apresentaram efeito significativo sobre a IPC, considerando-se $\mathrm{P}<0,0001$ e $\mathrm{P}<0,0001$ para o GSF e para o ANOP, respectivamente. A EP não apresentou diferença significativa.

Baruselli (1997) não observou diferenças regionais influenciando a IPC no país. Cassiano et al. (2003) observaram efeito significativo do ANOP na IPC, estando de acordo com os dados deste trabalho. No entanto, este efeito não pode ser atribuído à seleção do rebanho para IPC, visto que esta variou aleatoriamente durante os anos, não obedecendo a uma ordem crescente ou decrescente. 
Para o intervalo de partos, foram analisados 792 registros, distribuídos em seis raças e/ou grupos genéticos, abrangendo o período de 1983 a 2005. Nos trabalhos realizados por Baruselli (1993), a média do IDP foi próxima àquela obtida neste estudo, 375,6 $\pm 35,4$ dias $(12,11 \pm 1,16$ meses $)$, utilizando-se somente monta natural. Batista et al. (1994) obtiveram média para o IDP de 13,2 meses, tendo sido este valor o mais próximo daquele encontrado neste trabalho.

Rolim Filho (2006) avaliou 389 interpartos, em Belém, Pará. A média em relação à época de

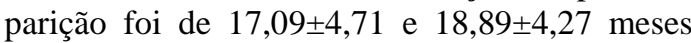
para a época mais chuvosa e menos chuvosa, respectivamente. $\mathrm{O}$ interparto médio encontrado por este autor foi de $16,54 \pm 3,74$ meses e valores mínimo e máximo de 11,33 e 28,31 meses.

Com base em 3.015 observações na região do Baixo Amazonas, Pará - Brasil, Cassiano et al. (2003) relataram IDP de aproximadamente 13 meses, tal qual observado neste trabalho.

A porcentagem de variação explicada pelo modelo proposto foi aproximadamente 6,6\% $\left(\mathrm{R}^{2}=0,06593\right)$. Isto se explica pelo fato de o IDP ser uma característica fortemente influenciada pelo manejo e/ou ambiente sobrecarregando o erro em virtude de causas de variação incontroláveis. Não foi observada influência significativa das fontes de variação (GSF, ANOP e EP) nos interpartos.

A exemplo deste estudo, Rolim Filho (2006) não encontrou influência significativa das fontes de variação no IDP. Para Vasconcellos (1996), a EP apresentou influência significativa, demonstrada pelo menor interparto dos animais nascidos na estação chuvosa.

De acordo com Zicarelli et al. (1997), vários fatores interferem na duração do IDP, entre os quais o fator nutricional, a idade da búfala, além de fatores ambientais e genéticos. Barkawi et al. (1998) encontraram interpartos menores na estação fria $(400,3 \pm 14,3$ dias $)$ e maiores na estação quente (441,5 $\pm 14,3$ dias).

Taneja (1999) relatou que o ano do parto foi altamente significativo em todas as características estudadas, incluindo o IDP. Sampaio Neto et al. (2001) verificaram que o IDP sofreu influência significativa do mês do parto anterior $(\mathrm{P}<0,01)$, o que também não foi encontrado neste trabalho.

Para a eficiência reprodutiva, foram estudados 587 registros em seis raças e/ou grupos genéticos, abrangendo o período de 1983 a 2005. Traad et al. (1991) relataram ER de aproximadamente $91,54 \%$, média muito próxima à encontrada neste estudo. Vários autores atribuem o manejo deficiente e/ou a seleção inadequada como fatores responsáveis pelos índices reprodutivos inferiores dos bubalinos em relação aos bovinos (Hafez, 1952, 1954, 1955; Vale, 1988; Vale et al., 1990; Baruselli, 1993; Vale, 2000).

Para Baruselli et al. (1995), somente será possível melhorar a eficiência reprodutiva e empregar biotecnologias quando requisitos básicos forem controlados, tais como: nutrição adequada, suplementação mineral correta, controle sanitário, controle produtivo e conduta dentro das recomendações técnicas.

Em búfalas, alguns autores consideram o intervalo de partos e a idade à primeira cria como os mais importantes parâmetros para se medir a eficiência reprodutiva (Sampaio Neto et al., 2001). Dentre os fatores que afetam a ER de rebanhos bubalinos, a detecção de cio é o que mais se destaca, devido à apresentação de sintomatologia discreta e à necessidade de pessoal mais capacitado para sua identificação (Baruselli et al., 1997; Zicarelli et al., 1997).

Rolim Filho (2006) verificou que animais em monta natural obtiveram maiores escores (118,08\%) para ER, portanto melhor eficiência, seguidos dos animais que utilizaram inseminação artificial em tempo fixo $(90,50 \%)$ e inseminação artificial (58,34\%). A média da ER foi $0,67 \pm 0,13$, com valores mínimo e máximo 0,50 e 0,93 , respectivamente.

A porcentagem de variação explicada pelo modelo proposto foi de aproximadamente $8,79 \%$ $\left(\mathrm{R}^{2}=0,0879\right)$. Neste estudo, não foi observada influência significativa das fontes de variação.

Bittencourt et al. (1997), ao estudarem a lucratividade dos sistemas de produção, verificaram que os mais lucrativos foram aqueles que obtiveram melhor relação entre desempenho reprodutivo e habilidade maternal da vaca. Segundo Schwengber et al. (2001), a fertilidade 
real não contempla as fêmeas que parem uma vez ou mesmo aquelas que não parem. Mercadante (1995) sugere precaução ao se avaliar a produtividade total, pois esta envolve muitas características de diferentes herdabilidades e importância econômica. Notter (1995) complementa afirmando que a herdabilidade dessas características tende a ser controlada pela característica mais variável e menos herdável.

O GSF e o ANOP mostraram influência significativa $\quad(\mathrm{P}<0,0001), \quad$ constituindo-se, portanto, de fontes de variação para essa característica. O modelo proposto foi capaz de explicar aproximadamente $21 \%$ das causas de variação $\left(R^{2}=0,2141\right)$.

Silveira et al. (2004) encontraram valor médio de $144 \mathrm{~kg}$ para fertilidade real e citam que houve influência significativa $(\mathrm{p}<0,01)$ dos efeitos fixos de ano e mês de nascimento pela interação ano do nascimento / mês do nascimento e pela ordem de parto.

McManus et al. (2002) encontraram valores inferiores a estes: $113,9 \mathrm{~kg}$ para vacas com bezerros e $111,85 \mathrm{~kg}$ para vacas com bezerras. Todavia, Campelo et al. (1999) obtiveram valor médio de $185 \mathrm{~kg}$. Ainda assim, nota-se alta variabilidade fenotípica nos resultados, indicando que a característica é complexa, pois envolve os eventos reprodutivos, a habilidade materna e a expressão dos genes de crescimento em uma única característica.

Ao calcularem a eficiência de produção de carne das vacas, Euclides Filho et al. (1992) encontraram valores de $2,18 \mathrm{~kg} / \mathrm{kg}^{(0,75)}$, $2,02 \mathrm{~kg} / \mathrm{kg}^{(0,75)}$ e $2,15 \mathrm{~kg} / \mathrm{kg}^{(0,75)}$, respectivamente, para os grupos Nelore-Fleckvieh, NeloreChianina e Nelore-Charolês para a produtividade ao primeiro parto.

Por outro lado, Alencar et al. (1997), ao compararem sistemas de cruzamentos entre raças de bovinos de corte, encontraram a média de $0,0693 \pm 0,0002 \mathrm{~kg}$ de bezerro ao parto $/ \mathrm{kg}$ de vaca ao parto e $0,427 \pm 0,002 \mathrm{~kg}$ de bezerro desmamado/kg de vaca ao parto.

Por conseguinte, Perotto et al. (2001) avaliaram a eficiência à desmama de 289 novilhas bovinas e obtiveram médias de $149 \pm 2 \mathrm{~kg}, 160 \pm 2 \mathrm{~kg}$, $172 \pm 1 \mathrm{~kg}, 166 \pm 2 \mathrm{~kg}$ e $188 \pm 1 \mathrm{~kg}$, respectivamente, para os grupos Nelore, 1/2 Guzerá + 1/2 Nelore, 1/2 Red Angus + 1/2 Nelore, 1/2 Marchigiana + $1 / 2$ Nelore e $1 / 2$ Simental $+1 / 2$ Nelore.

Ribeiro et al. (2001), por sua vez, utilizaram cinco meios para aferir a estimativa de eficiência produtiva de 62 vacas e encontraram, respectivamente, para o primeiro, segundo e terceiro resultados que variam de 34,6 a $42 \mathrm{~kg}$ de bezerro/100kg de vaca; 35,6 a $41,2 \mathrm{~kg}$ de bezerro/100kg de vaca ao desmame; e 5,85 a $6,60 \mathrm{~kg}$ de bezerro/kg de NDT da vaca. Para o quarto e quinto métodos, obtiveram 12,9 e $9,4 \%$ mais quilos de bezerros por unidade de peso metabólico, respectivamente.

Por fim, McManus et al. (2002) relataram a ocorrência dos valores médios do peso do bezerro ao nascer/peso da vaca ao parto e do peso do bezerro à desmama/peso da vaca ao parto, respectivamente, de $0,070 \pm 0,013 \mathrm{~kg}$ e $0,354 \pm 0,094 \mathrm{~kg}$.

Não existem dados sobre este índice em búfalos; neste estudo, somente o ano do parto mostrou influência significativa $(<0,0001)$ para produtividade ao primeiro parto. $\mathrm{O}$ modelo proposto foi capaz de explicar aproximadamente $33 \%$ das causas de variação $\left(R^{2}=0,3342\right)$.

Perotto et al. (2001) relataram que o grupo genético da novilha teve efeito significativo $(\mathrm{P}<0,001)$ sobre as características analisadas. Da mesma forma, o ano de nascimento da novilha influenciou $(\mathrm{P}<0,01)$ a idade à primeira parição e, consequentemente, o desempenho à desmama, assim como o ano de nascimento do bezerro, influenciando o peso deste à desmama, teve efeito $(\mathrm{P}<0,001) \quad$ sobre as características avaliadas.

Para a produtividade acumulada foram considerados os resultados obtidos em bovinos, dada a inexistência de relatos sobre este índice em búfalos. Segundo Lôbo (1996), a produtividade acumulada refere-se à contribuição da matriz durante sua permanência no rebanho, sendo dependente da idade ao primeiro parto e do intervalo de partos.

Schwengber et al. (2001) obtiveram média de $130 \pm 35 \mathrm{~kg}$ de bezerros desmamados por vaca ao ano, resultado inferior ao reportado por Rosa (1999), que foi $144 \mathrm{~kg}$. 
O GSF e o ano do parto mostraram influência significativa $(\mathrm{P}<0,0001)$, e o modelo proposto foi capaz de explicar aproximadamente $25 \%$ das causas de variação $\left(R^{2}=0,2548\right)$.

Para Schwengber et al. (2001), a fazenda, o ano de nascimento da vaca e o pai da vaca influenciam significativamente $(\mathrm{P}<0,0001)$ na produtividade acumulada. Rosa (1999), entretanto, não obteve o mesmo resultado; para ela, somente a fazenda e o ano de nascimento da vaca influenciaram a produtividade acumulada.

\section{CONCLUSÕES}

Búfalos das raças Murrah, Mediterrâneo, Jafarabadi, Tipo Baio, Carabao e seus produtos apresentaram, para as condições de pastagens nativas de terra inundável da Amazônia e terra firme, boa performance reprodutiva. Os índices de eficiência reprodutiva avaliados neste estudo mostraram-se dentro de uma variação aceitável indicando que há variabilidade genética para a produção de bubalinos nas condições estudadas. Nos estudos de avaliação e seleção dos animais, devem-se considerar a influência do ano do parto e o grau de sangue da fêmea. Os índices produtivos mostraram-se de grande valia para o melhoramento do rebanho, devendo ser mais estudados.

\section{REFERÊNCIAS}

ALENCAR, M.M.; TREMATORE, R.L.; OLIVEIRA, J.A.L. Desempenho produtivo de vacas da raça Nelore e cruzadas Charolês $x$ Nelore, Limousin x Nelore e Tabapuã x Gir. Rev. Bras. Zootec., v.26, p.467-472, 1997.

BARBOSA, N.G.S. Bubalinocultura no Estado do Pará. Rev. Bras. Reprod. Anim., v.29, p.34-38, 2005. Disponível em: www.cbra.org.br. Acessado em: 13/08/2012.

BARKAWI, A.H.; KHATTAB, R.M.; ELWARDANI, M.A. Reproductive efficiency of Egyptian buffaloes in relation to oestrus detection systems. Anim. Reprod. Sci., v.51, p.225-231, 1998.

BARUSELLI, P.S. Manejo reprodutivo de bubalinos. 1993. 46f. Monografia - Instituto de Zootecnia - Estação Experimental de Zootecnia do Vale do Ribeira. 1993.
BARUSELLI, P.S.; BARNABE, V.H.; BARNABE, R.C. et al. Condição corporal ao parto $\mathrm{e}$ eficiência reprodutiva de fêmeas bubalinas inseminadas artificialmente. In: IX CONGRESSO BRASILEIRO DE REPRODUÇÃO ANIMAL, 9., Belo Horizonte. 1995. Anais... Belo Horizonte: CBRA, 1995, v.1, p.380.

BARUSELLI, P.S. Reprodução de bubalinos. In: SIMPÓSIO BRASILEIRO DE BUBALINOCULTURA, 1., 1996, Cruz da Almas. Anais... Cruz das Almas: Universidade Federal da Bahia, 1997. p.117-153.

BARUSELLI, P.S.; MUCCIOLO, R.G.; VISINTIN, J.A. et al. Ovarian follicular dynamics during the estrous cycle in buffalo (Bubalus bubalis). Theriogenology, v.47, p.15311547, 1997.

BATISTA, H.A.M.; RODRIGUES FILHO, J.A.; LOURENÇO JÚNIOR, J.B. et al. Performance of mediterranean buffalos in cultivated pasture of the Amazon Region. In: VALE, W.G.; BARNABÉ, V.H.; MATTOS, J.C.A. (eds). WORLD BUFFALO CONGRESS, 4, 1994, São Paulo. Anais. São Paulo: ABCB/IBF/ FAO/FINEP, 1994. v.2, p.104-106.

BITTENCOURT, T.C.C.; LOBO, R.B.; REYES, A. de los et al. Uso da fertilidade real como medida de produtividade em rebanhos de bovinos de corte.In: O Nelore do século XXI, 4., 1997, Uberaba. Anais... Uberaba: ABCZ, 1997. p.242-244.

CAMARGO JÚNIOR, R.N.C. Estudo da eficiência reprodutiva de búfalos (Bubalus bubalis) na Amazônia Oriental. 2007. 48f. Dissertação (Mestrado em Ciência Animal) Universidade Federal do Pará, Universidade Federal Rural da Amazônia, Empresa Brasileira de Pesquisa Agropecuária, Belém, 2007.

CAMPELlO, C.C.; MARTINS FILHO, R.; LOBO, R.N.B. Intervalo de partos e fertilidade real em vacas nelore no estado do Maranhão. Rev. Bras. Zootec., v.28, p.474-479, 1999.

CASSIANO, L.A.P.; MARIANTE, A.S.; McMANUS, C. et al. Caracterização fenotípica de raças bubalinas nacionais e do tipo Baio. Pesq. Agrop. Bras., v.38, p.1337-1342, 2003. 
DE FRANCISCIS G. Buffalo improvement programme in Italy. In: SEMINAR SPONSORED BY FAO/SIDA/GOVT OF INDIA, 1978, India. Anais... India: FAO 1979. p.163-172.

EUCLIDES FILHO, K.; FIGUEIREDO, G.R.; THIAGO, L.R.L.S. Eficiência biológica de produção de carne de diferentes grupos genéticos. In: REUNIÃO ANUAL DA SOCIEDADE BRASILEIRA DE ZOOTECNIA. 29., 1992, Lavras. Anais... Lavras: SBZ, 1992. p.124.

HAFEZ, E.S.E. The buffaloes a review. Indian J. Vet. Sci., v.22, p.257-263, 1952.

HAFEZ, E.S.E. Oestrus and some related phenomena in the buffalo. J. Agricult. Sci., v.44, p.165-172, 1954.

HAFEZ, E.S.E. Puberty in buffalo-cow. $J$. Agricult. Sci., v.46, p.137-42, 1955.

IBGE. Diretoria de Pesquisas, Coordenação e Agropecuária, Pesquisa da Pecuária Municipal 2003. Disponível em <www.ibge.gov.br>. Acessado em: 25 out. 2005.

LÔBO, R.B. Programa de melhoramento genético da raça Nelore. 3.ed. Ribeirão Preto: FINEP, 1996. 104p.

McMANUS, C.M. SAUERESSIG, M.G.; FALCÃO, R.A. et al. Componentes reprodutivos e produtivos no rebanho mestiço de corte da Embrapa Cerrados. Rev. Bras. Zootec., v.31, p.648-657, 2002.

MARQUES, J.R.F. BATISTA, H.A.M.; NASCIMENTO, C.N.B. et al. Idade à primeira cria em bubalinos no trópico úmido brasileiro. Belém: Embrapa-CPATU, 1985. 13p. (Boletim de Pesquisa, 70). Disponível em: http://www.infoteca.cnptia.embrapa.br/handle/do c/380954. Acessado em: 13/08/2012.

MARQUES, J.R.F. Avaliação genéticoquantitativa de algumas características do desempenho produtivo de grupos genéticos de búfalos (Bubalus bubalis L.). 1991. 148f. Tese (Doutorado em Zootecnia) - Universidade Estadual Paulista, 1991.
MERCADANTE, M.E.Z. Estudo das relações genéticoquantitativas entre características de reprodução, crescimento e produção em fêmeas da raça Nelore. 1995. 108f. Dissertação (Mestrado em Genética) - Faculdade de Medicina de Ribeirão Preto/Universidade de São Paulo, 1995.

NOTTER, D.R. Maximizing fertility in animal breeding programs. In: CONGRESSO BRASILEIRO DE REPRODUÇÃO ANIMAL, 11., 1995, Belo Horizonte. Anais... Belo Horizonte: Colégio Brasileiro de Reprodução Animal, 1995. 36p.

PEROTTO, D.; ABRAHÃO, J.J.S.; KROETZ, I.A. Produtividade à desmama de novilhas Nelore e F1 Bos taurus x Nelore e Bos indicus x Nelore. Rev. Bras. Zootec. v.30, p.1712-1719, 2001. Disponível em: http://www.revista.sbz.org.br/ artigo/index.php?artigo=3114. Acessado em: 13/08/2012.

RIBEIRO, E.L.A.; RESTLE, J.; ROCHA, M. A.; et al. Eficiência produtiva em vacas primíparas das raças Aberdeen Angus e Charolês. Rev. Bras. Zootec. v.30, p.125-132, 2001. Disponível em: http://www.scielo.br/pdf/rbz/v30n1/5444.pdf.

Acessado em: 13/08/2012.

ROLIM FILHO, S.T. Aspectos da eficiência reprodutiva de bubalinos criados em sistema misto (várzea e terra firme) em Belém. 2006. 87f. Dissertação (Mestrado) - Curso de PósGraduação em Ciência Animal, Universidade Federal do Pará, Universidade Federal Rural da Amazônia, Empresa Brasileira de Pesquisa Agropecuária, 2006.

ROSA, A.N. Variabilidade fenotípica e genética do peso adulto e da produtividade acumulada de matrizes em rebanhos de seleção da raça Nelore no Brasil. 1999, 114f. Tese (Doutorado em Genética) - Faculdade de Medicina de Ribeirão Preto/Universidade de São Paulo, 1999.

SAMPAIO NETO, J.C.; MARTINS FILHO, R.; LÔBO, R.N.B. et al. Avaliação dos Desempenhos Produtivo e Reprodutivo de um Rebanho Bubalino no estado do Ceará. Rev. Bras. Zootec., v.30, p.368-373, 2001. Disponível em: http://www.scielo.br/pdf/rbz/v30n2/5476.pdf. Acessado em: 13/08/2012. 
STATISTICAL Analysis System. SAS Institute Inc. SAS Introductory Guide for Personal Computer's, Version 8, Cary, N.C. USA, 2002.

SCHWENGBER, E.B.; BEZERRA, L.A.F.; LOBO, R.B. Produtividade acumulada como critério de seleção em fêmeas da raça nelore. Cienc. Rural [on line]. v.31, p.483-486. 2001. Disponível em: http://www.scielo.br/pdf/cr/v31n3/ a20v31n3.pdf. Acessado em: 13/08/2012.

SILVEIRA, J.C.; McMANUS, C.; MASCIOLI, A.S. et al. Fatores ambientais e parâmetros genéticos para características produtivas $\mathrm{e}$ reprodutivas em um rebanho nelore no estado do Mato Grosso do Sul. Rev. Bras. Zootec., v.33, p.1432-1444, 2004. Disponível em: http://www.scielo.br/scielo.php?pid=s151635982004000600010\&script=sci_arttext. Acessado em: 13/08/2012.

TANEJA, V.K. Dairy breeds and selection. In: FALVEY, L.; CHANTALAKHANA, C. (Ed.). Smallholder dairying in the Tropics. Nairobi: International Livestock Research Institute, 1999, p.462.

TRAAD DA SILVA, M.E.; PINTO, J.M.; KROETZ, I.A. et al. Performance of a Murrah buffalo production system in the Paraná stateBrazil. In: WORLD BUFFALO CONGRESS, 3 ., 1991, Varna. Anais... Varna: [s.n.] 1991, v.1, p.28.
VALE, W.G. Bubalinos: fisiologia e patologia da reprodução. Campinas: Fundação Cargill. 1988. p.86.

VALE, W.G.; OHASHI, O. M.; SOUSAY, J.S. et al. Studies on the reproduction of water buffalo in the Amazon basin. Livestock in Latin America. Vienna: International Atomic Energy Agency, 1990. p.201-210.

VALE, W.G. Enhancing the puberty in buffalo heifers. In: INTERNATIONAL CONGRESS ON ANIMAL REPRODUCTION, 14., 2000, Stockholm. Anais... Stockholm: ICAR, 2000. v.1, p.271.

VASCONCELLOS, B.F. Estudo da endogamia em um rebanho bubalino da raça Murrah. Jaboticabal. 1996. 69f. Dissertação (Mestrado em Zootecnia) - Universidade Estadual Paulista, 1996.

ZICARELLI, L.; DE FILIPPO, C.; FRANCILLO, M. et al. Influence of insemination technique and ovulation time on fertility percentage in synchronized buffaloes. In: WORLD BUFFALO CONGRESS, 5, 1997, Caserta. Anais... Caserta: [s.n.], 1997. p.732-737. 